\title{
BUTTERFLY-ORTHOGONAL LOOP SUBDIVISION WAVELETS
}

\author{
Huawei Wang ${ }^{1,2,3}$, Guiming Qin ${ }^{2,3}, \mathrm{Li} \mathrm{Xiao}^{2,3}$ and $\mathrm{Yi} \mathrm{Cao}^{2,3}$ \\ ${ }^{1}$ Laboratory of Computational Physics, 6 Huayuan Road, Haidian, Beijing 100088, China \\ ${ }^{2}$ Institute of Applied Physics and Computational Mathematics, 2 Fenghao East Road, Haidian, Beijing 100094, China \\ ${ }^{3}$ CAEP Software Center for High Performance Numerical Simulation, 6 Huayuan Road, Haidian, Beijing 100088, China
}

\begin{abstract}
In this paper, we have proposed a new butterfly-orthogonal Loop subdivision wavelet based on the lifting scheme. We use a butterfly-style local orthogonalization structure to optimize the free parameters introduced in the wavelet transforms so as to obtain a very stable and nearly semi-orthogonal wavelet. Although it takes a little more computation time than the existing Loop subdivision wavelets, the proposed wavelet possesses a better fitting quality as well as performance of denoising, as demonstrated in the experiments. Similar to the other Loop subdivision wavelets, the butterfly-orthogonal wavelet has a linear computing complexity, because all lifting operations in the wavelet transforms are of perfect in-place computing and relate to only local vertex points or edge points. The proposed wavelet analysis can be used in a wide range of applications, including progressive transmission, shape approximation, data compression and multiresolution rendering.
\end{abstract}

\section{KEYWORDS}

Wavelet Analysis, Butterfly-Orthogonalization, Loop Subdivision Wavelet, Lifting Scheme

\section{INTRODUCTION}

Multiresolution modeling techniques attract much attention in recent decades, because they can address the challenge of modeling massive polygons obtained by high-resolution 3D scanning and have a wide range of applications such as progressive transmission, data compression, level-of-detail control and multiresolution rendering. Among multiresolution techniques, wavelet is a commonly-used tool, and subdivision is another powerful tool for representing smooth surfaces of arbitrary topology in multiple resolutions. By combining them naturally, people have focused on subdivision-based wavelet analyses for efficiently representing highly-detailed geometric models. The key to constructing such wavelets is to decompose the subdivision rules into a series of reversible local operations and then convert the forward and inverse subdivisions into wavelet transforms. Usually, the reconstruction and decomposition algorithms of subdivision-based wavelets are very simple, quite efficient and practical.

Subdivisions and wavelets are joined smoothly by the lifting scheme (Schröder and Sweldens, 1995; Sweldens, 1996), which is an efficient technique for constructing second-generation wavelets. A simple wavelet, e.g. a lazy wavelet, can be improved according to users' design requests by several steps of lifting. Schröder and Sweldens showed an example of constructing lifting wavelets on the sphere with customized properties (Schröder and Sweldens, 1995). Lounsbery et al. proposed a class of wavelets based on subdivision surfaces so as to generalize wavelet analysis to functions defined on compact surfaces of arbitrary topology (Lounsbery et al., 1997). Based on the hierarchical Delaunay triangulation, a multiresolution analysis on planar or spherical meshes of irregular connectivity is proposed in (Bonneau, 1998). By generalizing the uniform subdivision to a new irregular subdivision scheme in topology, Valette and Prost extended the work of Lounsbery et al. and proposed a wavelet-based multiresolution analysis that can be applied directly to irregular meshes, thus the connectivity of the processed mesh keeps unchanged (Valette and Prost, 2004).

Samavati and Bartels showed how to reverse subdivision rules and construct the wavelets by straightforward matrix observations (Samavati and Bartels, 1999). By using a quadrature mirror construction, Khodakovsky et al. constructed a Loop wavelet transform (Khodakovsky et al., 2000). Samavati et al. proposed multiresolution surfaces of arbitrary topology by locally reversing the Doo subdivision scheme (Samavati et 
al., 2002; Doo and Sabin, 1978). Wu and Amaratunga used the wavelets based on the butterfly scheme (Zorin et al., 1996) to deal with GIS data (Wu and Amaratunga, 2003). Using local lifting operations performed on polygonal meshes, Bertram et al. gave a new construction of lifted biorthogonal wavelets on surfaces of arbitrary two-manifold topology (Catmull and Clark, 1978) and presented generalized B-spline subdivision surface wavelets (Bertram et al., 2004). Based on local orthogonalization using discrete inner products, Bertram presented a biorthogonal wavelet construction for Loop subdivision (Bertram, 2004). Li et al. independently proposed another unlifted Loop subdivision wavelets by optimizing free parameters in the extended subdivision steps (Li et al., 2004). Later, Wang et al. proposed an effective wavelet construction for general Catmull-Clark subdivisions (Wang et al., 2006), and the resulting wavelets have better fitting quality than the existing Catmull-Clark-like subdivision wavelets (Bertram et al., 2000, 2004). Such wavelet construction methods based on local lifting operators are very efficient, e.g., usually in linear-time complexity as well as fully in-place operations, and thus do not need to solve global sparse linear systems.

In order to improve the stability of Loop subdivision wavelets, we present a new Butterfly-orthogonal wavelet in the paper by using the lifting scheme. Compared with the previous Loop subdivision wavelets, the wavelet has better stability as well as fitting quality. The resulting wavelet transforms still have a linear computational complexity because they are composed of local and in-place lifting operations. The wavelet analysis can be used in a variety of applications such as progressive transmission, data compression, shape approximation and real-time multiresolution rendering.

\section{LOOP SUBDIVISION}

Subdivision is attractive due to its powerful ability of modeling smooth surfaces of arbitrary topology as well as its multi-resolution representation format. Loop subdivision is designed to generalize the recurrence relations for the three-directional quartic box splines to triangular meshes of arbitrary topology (Loop, 1987). As triangular meshes arise in many applications, Loop subdivision becomes one of the most popular subdivision schemes. A Loop surface is defined as the limit of a sequence of finer and finer meshes produced in the recursive subdivision of an initial control mesh.
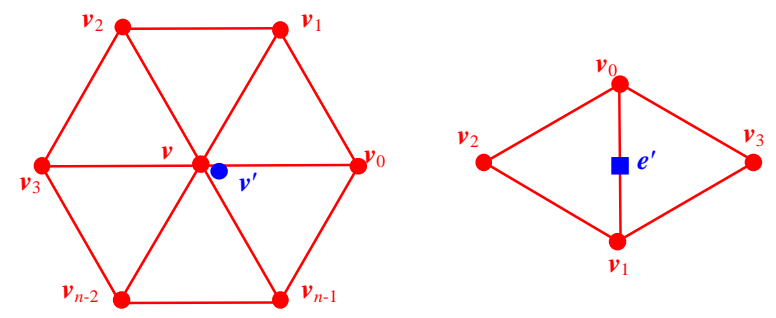

Figure 1. Local structures: (Left) updating an old vertex; (Right) computing the new vertex for an edge

In a refinement step, a triangle is divided into four pieces by updating every old vertex and introducing a new vertex for each edge. The local structures of control vertices for updating an old vertex and computing the new vertex for an edge are shown in Figure 1, and the corresponding computing formulae are given as follows:

$$
\boldsymbol{v}^{\prime}=a(n) \boldsymbol{v}+b(n) \sum_{i=0}^{n-1} \boldsymbol{v}_{i}, e^{\prime}=\frac{3}{8}\left(\boldsymbol{v}_{0}+\boldsymbol{v}_{1}\right)+\frac{1}{8}\left(\boldsymbol{v}_{2}+\boldsymbol{v}_{3}\right),
$$

where $n \geq 3$ is the valence of $v$ and

$$
a(n)=\frac{3}{8}+\frac{(3+2 \cos (2 \pi / n))^{2}}{64}, b(n)=\frac{1-a(n)}{n} .
$$

$\boldsymbol{v}^{\prime}$ and $\boldsymbol{e}^{\prime}$ are usually called the vertex point and the edge point, respectively. Each vertex point has the same valence as the corresponding old vertex while all edge points are of valence 6 . A vertex with valence other than 6 is regarded as an extraordinary point. After one step of refinement, all extraordinary points will be isolated. 


\section{IMPROVED SUBDIVISION OPERATIONS AND WAVELETS}

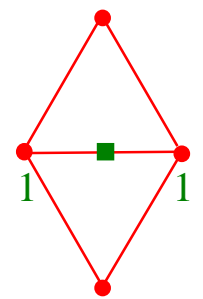

(a) $v \triangleright e$

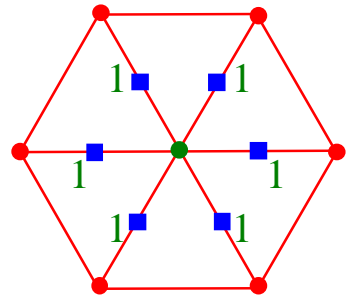

(b) $e \triangleright v$

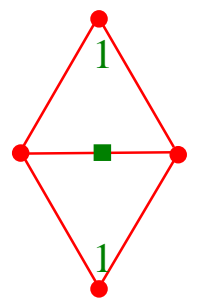

(c) $s \triangleright \boldsymbol{e}$

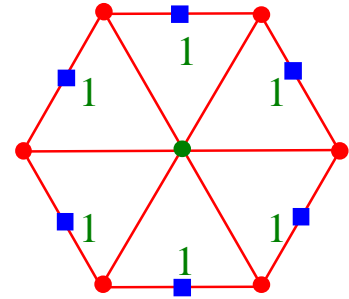

(d) $e \triangleright s$

Figure 2. Un-normalized masks for local operators

The key idea for constructing subdivision-based wavelets is to decompose a complicated wavelet transform into a series of reversible simple local operations (also called lifting operations). The four local operators used in (Li et al., 2004) are listed in Figure 2, where the operator $\boldsymbol{v} \triangleright \boldsymbol{e}$ means "take the average of the two vertex points adjacent to an edge point", $s \triangleright \boldsymbol{e}$ means "take the average of the two sideling points adjacent to an edge point", the operator $\boldsymbol{e} \triangleright \boldsymbol{v}$ means likewise "take the average of the edge points adjacent to a vertex point" and $\boldsymbol{e}$ $\triangleright s$ is defined similarly. Using these local operators, the rules of Loop subdivision (1) can be expressed equivalently as the following two steps of local operations:

$$
\begin{aligned}
& \text { i) } \boldsymbol{e} \leftarrow \frac{3}{4}(\boldsymbol{v} \triangleright \boldsymbol{e})+\frac{1}{4}(\boldsymbol{s} \triangleright \boldsymbol{e}), \\
& \text { ii) } \quad \boldsymbol{v} \leftarrow \alpha \boldsymbol{v}+n \beta(\boldsymbol{e} \triangleright \boldsymbol{v}),
\end{aligned}
$$

where $\alpha=\alpha(n)=[8 a(n)-3] / 5$ and $\beta=\beta(n)=[1-\alpha(n)] / n$. By introducing a vector $\boldsymbol{e}$ for each edge point initially and then adding an additional lifting operation, one can extend the above subdivision (2) into an in-place reversible transform ( $\mathrm{Li}$ et al., 2004):

$$
\begin{array}{ll}
\text { i) } & \boldsymbol{v} \leftarrow \boldsymbol{v}+\eta_{0}(\boldsymbol{e} \triangleright \boldsymbol{v})+\eta_{1}(\boldsymbol{e} \triangleright \boldsymbol{s}), \\
\text { ii) } & \boldsymbol{e} \leftarrow \gamma \boldsymbol{e}+\frac{3}{4}(\boldsymbol{v} \triangleright \boldsymbol{e})+\frac{1}{4}(\boldsymbol{s} \triangleright \boldsymbol{e}), \\
\text { iii) } & \boldsymbol{v} \leftarrow \alpha \boldsymbol{v}+n \beta(\boldsymbol{e} \triangleright \boldsymbol{v}),
\end{array}
$$

where $\eta_{0}, \eta_{1}$ and $\gamma(\gamma \neq 0)$ are free parameters and can be adjusted later. Note that the forward subdivision (3) is equivalent to the original Loop subdivision expressed by Eq. (1) or (2) when all $\boldsymbol{e}$ are initially equal to $\boldsymbol{0}$. Since each step is reversible in Equation (3), by inverting the steps and arranging them in the reverse order, we have

$$
\begin{array}{ll}
\text { i) } & \boldsymbol{v} \leftarrow[\boldsymbol{v}-n \beta(\boldsymbol{e} \triangleright \boldsymbol{v})] / \alpha, \\
\text { ii) } & \boldsymbol{e} \leftarrow\left[\boldsymbol{e}-\frac{3}{4}(\boldsymbol{v} \triangleright \boldsymbol{e})-\frac{1}{4}(\boldsymbol{s} \triangleright \boldsymbol{e})\right] / \gamma, \\
\text { iii) } & \boldsymbol{v} \leftarrow \boldsymbol{v}-\eta_{0}(\boldsymbol{e} \triangleright \boldsymbol{v})-\eta_{1}(\boldsymbol{e} \triangleright \boldsymbol{s}) .
\end{array}
$$

Equations (3) and (4) are forward and inverse subdivision processes, respectively, which actually constitute the multiresolution synthesis and analysis framework of a Loop subdivision wavelet (Li et al., 2004). The initial vectors $\boldsymbol{e}$ can be regarded as wavelet coefficients accordingly. They will contribute to the resulting limit surface as surface details at each resolution level.

In order to achieve better stability, we will improve the construction of the Loop subdivision wavelet in two aspects. At first, we will introduce a new local operator $\boldsymbol{e} \triangleright \boldsymbol{f}$, which means "take the average of the farther-sideling edge points adjacent to a vertex point", as shown in Figure 3. The forward subdivision is then improved as

$$
\begin{array}{ll}
\text { i) } & \boldsymbol{v} \leftarrow \boldsymbol{v}+\eta_{0}(\boldsymbol{e} \triangleright \boldsymbol{v})+\eta_{1}(\boldsymbol{e} \triangleright \boldsymbol{s})+\eta_{2}(\boldsymbol{e} \triangleright \boldsymbol{f}), \\
\text { ii) } & \boldsymbol{e} \leftarrow \boldsymbol{e}+\frac{3}{4}(\boldsymbol{v} \triangleright \boldsymbol{e})+\frac{1}{4}(\boldsymbol{s} \triangleright \boldsymbol{e}), \\
\text { iii) } & \boldsymbol{v} \leftarrow \alpha \boldsymbol{v}+n \beta(\boldsymbol{e} \triangleright \boldsymbol{v}),
\end{array}
$$

where the free parameter $\gamma$ is omitted because it is usually set to 1 as shown in (Li et al., 2004). Afterwards, we will turn the three free parameters in the first step of (5) into more free parameters so that we have more degrees of freedom to optimize the resulting wavelet. We will assign a distinct coefficient respectively for each of the $4 n$ edge points instead of one for each type of edge points. Therefore, we number the $n$ adjacent edge 
points, the $n$ sideling edge points and the $2 n$ farther-sideling edge points as $0,1, \ldots, n-1, \ldots, 2 n-1, \ldots, 4 n-1$, and the coefficient for the edge point $\boldsymbol{e}_{i}$ is denoted by $\omega\left(\boldsymbol{v}, \boldsymbol{e}_{i}\right)$. Note that $\omega\left(\boldsymbol{v}, \boldsymbol{e}_{i}\right)$ depends on the connection relation of local vertices around $\boldsymbol{v}$ and $\boldsymbol{e}_{i}$. Thus, we can finally rewrite the improved subdivision as

and its inverse is given as follows:

$$
\begin{array}{ll}
\text { i) } & \boldsymbol{v} \leftarrow \boldsymbol{v}+\sum_{i=0}^{4 n-1} \omega\left(\boldsymbol{v}, \boldsymbol{e}_{i}\right) \boldsymbol{e}_{i}, \\
\text { ii) } & \boldsymbol{e} \leftarrow \boldsymbol{e}+\frac{3}{4}(\boldsymbol{v} \triangleright \boldsymbol{e})+\frac{1}{4}(\boldsymbol{s} \triangleright \boldsymbol{e}), \\
\text { iii) } & \boldsymbol{v} \leftarrow \alpha \boldsymbol{v}+n \beta(\boldsymbol{e} \triangleright \boldsymbol{v}),
\end{array}
$$

$$
\begin{array}{ll}
\text { i) } & \boldsymbol{v} \leftarrow[\boldsymbol{v}-n \beta(\boldsymbol{e} \triangleright \boldsymbol{v})] / \alpha, \\
\text { ii) } & \boldsymbol{e} \leftarrow \boldsymbol{e}-\frac{3}{4}(\boldsymbol{v} \triangleright \boldsymbol{e})-\frac{1}{4}(\boldsymbol{s} \triangleright \boldsymbol{e}), \\
\text { iii) } & \boldsymbol{v} \leftarrow \boldsymbol{v}-\sum_{i=0}^{4 n-1} \omega\left(\boldsymbol{v}, \boldsymbol{e}_{i}\right) \boldsymbol{e}_{i} .
\end{array}
$$

Obviously, all lifting operations in the forward subdivision (6) and its inverse (7) are of perfect in-place computations, and relate to only the local vertex points or edge points, so the computations of wavelet synthesis and analysis are very efficient.

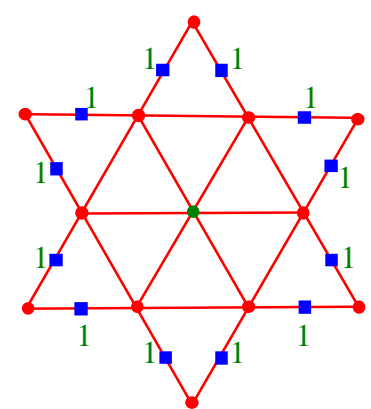

Figure 3. Un-normalized mask for a new local operator

\section{OPTIMIZING THE FREE PARAMETERS}

In the section, we will choose proper free parameters to optimize the performance of the resulting wavelet analysis. From the point of view of data reduction, the orthogonality of wavelets with scaling functions is a main goal of custom design of lifted wavelets to eliminate the data correlation. It is known that the last two steps of the forward subdivision (6) define a lazy wavelet synthesis (Bertram, 2004), and the basis functions corresponding to $\boldsymbol{e}$ (lazy wavelet $\psi$ ) and $\boldsymbol{v}$ (scaling function $\phi$ ) are defined naturally by the two steps of lifting operations. The first lifting operation in (6) is equivalent to a lifting step of the lay wavelet as follows:

$$
\psi^{\prime}=\psi+\sum_{k=0}^{7} \omega\left(\boldsymbol{v}_{k}, \boldsymbol{e}\right) \phi_{k},
$$

and the local situation of the wavelet function and related scaling functions is shown in Figure 4. Since the final wavelet function is $\psi^{\prime}$ after the lifting (8) and the scaling functions are not changed, the eight free parameters in (8) may be used to force the following eight orthogonality constraints:

$$
<\phi_{k}, \psi^{\prime}>=0, k=0,1, \cdots, 7,
$$

By substituting Eq. (8) into (9), we can easily obtain the eight parameters:

$$
\left[\omega\left(\boldsymbol{v}_{0}, \boldsymbol{e}\right), \omega\left(\boldsymbol{v}_{1}, \boldsymbol{e}\right), \cdots, \omega\left(\boldsymbol{v}_{7}, \boldsymbol{e}\right)\right]^{\mathrm{T}}=\boldsymbol{A}^{-1} \boldsymbol{B},
$$

where

$$
\boldsymbol{A}=\left(A_{i, j}\right)_{8 \times 8}=\left(<\phi_{i}, \phi_{j}>\right)_{8 \times 8}, \boldsymbol{B}=-\left(<\phi_{0}, \psi>,<\phi_{1}, \psi>, \cdots,<\phi_{7}, \psi>\right)^{\mathrm{T}} .
$$

The above computing process actually corresponds to a local orthogonalization (Lounsbery et al., 1997, Bertram, 2004) of the lazy wavelet, which will produce a nearly semi-orthogonal wavelet. The more scaling functions the wavelet orthogonalizes with, the more close to the global semi-orthogonal one the wavelet transform is. The local structure used in the orthogonalization is just like a butterfly, as shown in Figure 4, thus the resulting new wavelet is called the butterfly-orthogonal Loop subdivision wavelet in the paper. 


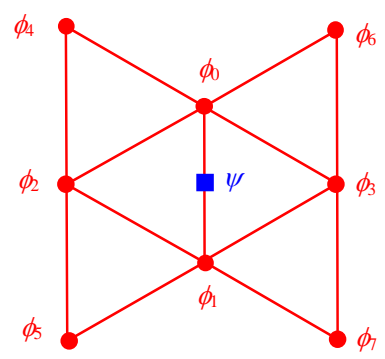

Figure 4. Local butterfly structure for orthogonalizing a lazy wavelet

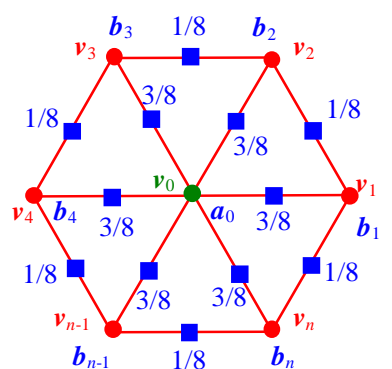

(b)

Figure 5. Discrete basis functions: a) wavelet $\psi ;$ b) scaling function $\phi_{0}$

Next, we will discuss the computation of the inner products of basis functions in the matrices $\boldsymbol{A}$ and $\boldsymbol{B}$. In order to enhance the efficiency of wavelet transforms, we do not use the continuous shape of wavelets and scaling functions directly but adopt a discrete inner product operating on the subdivision masks (Bertram, 2004; Samavati and Bartels, 1999). As shown in Figure 5, the discrete masks of lazy wavelets and scaling functions can be obtained from the subdivision formulae in the last two steps of Eq. (6) and are exactly the same as those presented in (Bertram, 2004). Here, $a\left(n_{i}\right)$ is simplified as $a_{i}$ where $n_{i}$ denotes the valence of the vertex $\boldsymbol{v}_{i}$, and $b_{i}, \alpha_{i}$ and $\beta_{i}$ are defined analogously. If $n$ is $6, a(n)$ is simplified further as $a$ for short, and $b(6), \alpha(6)$ and $\beta(6)$ are abbreviated similarly. Then, if supposing all nearby vertices other than $v_{0} \sim v_{7}$ to be regular and the local structure to be nondegenerative, we can deduce the following formulae:

$$
\begin{aligned}
& A_{i, i}=\left\{\begin{array}{c}
\frac{5}{32} n_{i}+a_{i}^{2}+b_{1-i}{ }^{2}+b_{2}{ }^{2}+b_{3}{ }^{2}+b_{4+i}{ }^{2} \\
+b_{6+i}{ }^{2}+\left(n_{i}-5\right) b^{2}, \text { if } n_{i}>4, \\
\frac{5}{32} n_{i}+a_{i}{ }^{2}+b_{1-i}{ }^{2}+b_{2}{ }^{2}+b_{3}{ }^{2}+\left(1-\delta_{i}\right) b_{4+i}{ }^{2}, \text { otherwise, }
\end{array}\right. \\
& A_{2 j, 2 j+1}=\left\{\begin{aligned}
& \frac{21}{64}+a_{2 j} b_{2 j}+a_{2 j+1} b_{2 j+1}+b_{j}^{2}+b^{2}, \text { if } n_{j}=4, \\
& \delta_{j} A_{2 j, 2 j}+\pi_{j}\left(\frac{1}{64}+b_{j}^{2}+b^{2}\right) \\
& \quad+\left(1-\delta_{j}\right)\left(1-\pi_{j}\right) b_{j}^{2}, \text { otherwise, }
\end{aligned}\right. \\
& A_{2+i, 2+i}=\frac{5}{32} n_{2+i}+a_{2+i}^{2}+b_{0}^{2}+b_{1}^{2}+b_{4+2 i}^{2} \\
& +\left(1-\delta_{2+i}\right)\left[b_{5+2 i}^{2}+\left(n_{2+i}-4\right) b^{2}\right], \\
& A_{2 j+i, 2 j+i}=\frac{5}{32} n_{2 j+i}+a_{2 j+i}^{2}+b_{i}^{2}+b_{j}^{2}+\left(n_{2 j+i}-2\right) b^{2} \\
& +\pi_{i}\left(b_{2(5-j)+i}{ }^{2}-b^{2}\right)+\chi_{i}\left(b_{5-j}{ }^{2}-b^{2}\right) \\
& +\chi_{j}\left(b_{2 j+1-i}{ }^{2}-b^{2}\right)+\delta_{j}\left(b_{1-i}{ }^{2}-b^{2}\right), \\
& A_{0,1}=\frac{21}{64}+a_{0} b_{0}+a_{1} b_{1}+b_{2}{ }^{2}+b_{3}{ }^{2}+\delta_{2}\left(\frac{1}{64}+b_{4}{ }^{2}\right)+\delta_{3}\left(\frac{1}{64}+b_{6}{ }^{2}\right] \text {, } \\
& A_{i, j}=\frac{21}{64}+a_{i} b_{i}+a_{j} b_{j}+b_{1-i}{ }^{2}+b_{2 j+i}{ }^{2}+\delta_{1-i}\left(\frac{1}{64}+b_{5-j}{ }^{2}\right) \\
& +\delta_{2 j+i}\left[\frac{1}{64}+b^{2}+\pi_{i}\left(b_{2(5-j)+i}{ }^{2}-b^{2}\right)\right. \\
& \left.+\chi_{i}\left(b_{5-j}{ }^{2}-b^{2}\right)+\chi_{j}\left(b_{2 j+1-i}{ }^{2}-b^{2}\right)-\delta_{j} b^{2}\right], \\
& A_{i, 2 j+i}=\frac{21}{64}+a_{i} b_{i}+a_{2 j+i} b_{2 j+i}+b_{j}{ }^{2}+b^{2}+\delta_{j}\left(\frac{1}{64}+b_{1-i}{ }^{2}\right) \\
& +\pi_{i}\left[b_{2(5-j)+i}{ }^{2}-b^{2}+\delta_{2(5-j)+i}\left(\frac{1}{64}+b_{5-j}{ }^{2}\right)\right] \\
& +\chi_{i}\left[b_{5-j}{ }^{2}-b^{2}+\delta_{5-j}\left(\frac{1}{64}+b_{1-i}{ }^{2}\right)\right], \\
& A_{i, 2 j+1-i}=\left\{\begin{array}{l}
A_{i, 2 j+i}, \text { if } n_{j}=3, \\
\frac{1}{64}+b_{1-i}{ }^{2}+b_{j}{ }^{2}+\chi_{1-i}\left(\frac{1}{64}+b_{5-j}{ }^{2}\right)+\chi_{j}\left(\frac{1}{64}+b_{2 j+i}{ }^{2}\right),
\end{array}\right. \\
& A_{j, 2 j+i}=\frac{21}{64}+a_{j} b_{j}+a_{2 j+i} b_{2 j+i}+b_{i}^{2}+b^{2}+\chi_{j}\left(b_{2 j+1-i}{ }^{2}-b^{2}\right) \text {, } \\
& A_{5-j, 2 j+i}=\left(1-\chi_{i}\right) b_{i}^{2}+\chi_{i} A_{5-j, 2(5-j)+i} \text {, } \\
& A_{2,3}=\frac{1}{64}+b_{0}^{2}+b_{1}^{2}+\chi_{0}\left(\frac{1}{64}+b_{4}^{2}\right)+\chi_{1}\left(\frac{1}{64}+b_{5}^{2}\right) \\
& +\left(\delta_{0}+\delta_{1}-\delta_{0} \delta_{1}\right)\left(\frac{21}{64}+a_{2} b_{2}+a_{3} b_{3}+b^{2}\right)-\delta_{0} \delta_{1} b^{2},
\end{aligned}
$$


$\forall i \in\{0,1\}, \forall j \in\{2,3\}$, where $\delta_{k} \equiv \delta\left(n_{k}\right)$ is equal to 1 if $n_{k}=3$ and 0 otherwise; $\chi_{k} \equiv \chi\left(n_{k}\right)$ is equal to 1 if $n_{k}=4$ and 0 otherwise; $\pi_{k} \equiv \pi\left(n_{k}\right)$ is equal to 1 if $n_{k}=5$ and 0 otherwise.

As a result, we can obtain all free parameters by computing the formula (10) after the matrices $\boldsymbol{A}$ and $\boldsymbol{B}$ are computed, and thus we obtain a new butterfly-style wavelet analysis based on Loop subdivision, which has an in-place and linear-time computation. Since extraordinary points are enough far from each other after two subdivision steps, there is at most one extraordinary point in the local structure shown in Figure 4. Therefore, in order to enhance the real-time computing efficiency, we can pre-compute the free parameters for the cases of the local structure containing at most one extraordinary point.

\section{EXPERIMENTAL RESULTS}

We will carry out the noise-filtering experiment (Bertram, 2004; Bertram et al., 2004) to check the stability of the wavelet proposed in the paper. Firstly, we subdivide a triangular mesh six times by the original Loop subdivision rules (1), and then add white noise to the vertices of the resulting mesh. The mesh obtained finally can be regarded as the fully detailed model, which will be analyzed step by step using the proposed wavelet analysis algorithm (7). The noise-filtered versions of the model at each level are shown in Figure 6 from level 6 to level 0 , respectively. It is obvious that the wavelet analysis has a very good stability because the influence of the white noise on the model decreases gradually with the increase of the filtering time.
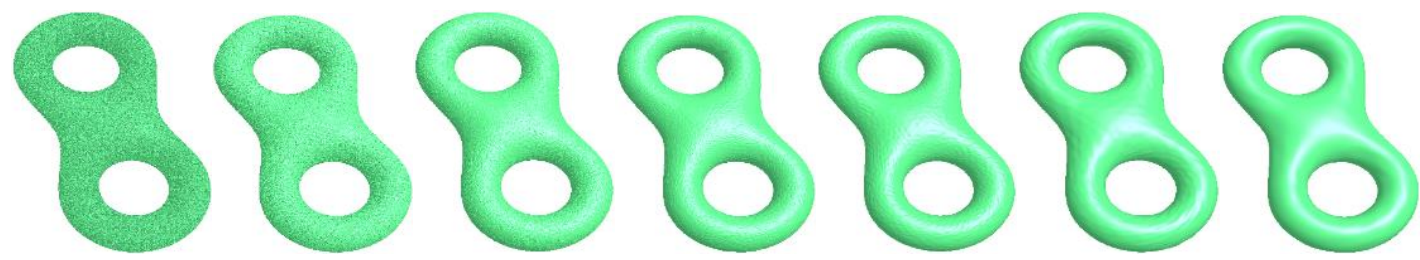

Figure 6. Wavelet analysis from level 6 to level 0

(a)

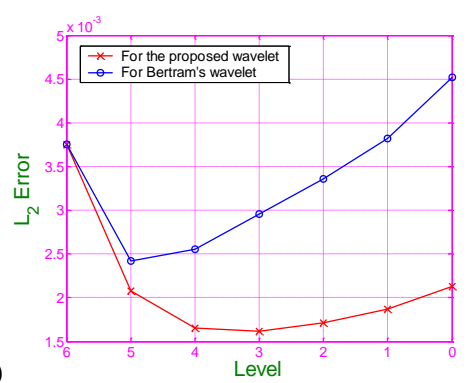

Figure 7. Sketch maps of (a) noisy errors and (b) computation times varying with levels

Furthermore, we have computed the $\mathrm{L}_{2}$ norm error of each low-pass filtered noisy mesh with respect to the undisturbed model. The errors for Bertram's Loop subdivision wavelet (Bertram, 2004) are also computed for comparison in the condition of the same model and the same noise. The varying sketches of the errors with regard to the levels are shown in Figure 7(a) for the two wavelets, respectively. It is shown that the noisy error for the proposed wavelet is always less than that for Bertram's wavelet at each level and actually equal to about a half of that for Bertram's wavelet after three times of filtering. The overall denoising ratio, or the ratio of the noisy error at level 0 to the initial error, is equal to 0.5680 for the proposed wavelet, which is obviously smaller than the overall denoising ratio 1.2053 for Bertram's wavelet. Thus, compared with Bertram's wavelet, the wavelet proposed in the paper has a better stability as well as a better performance of noise reduction.

In addition, we have also recorded the computation times when the wavelet transforms are performed. The platform is a PC equipped with a $2.8 \mathrm{GHz}$ Pentium $4 \mathrm{CPU}$ and $512 \mathrm{MB}$ RAM and running Windows XP Professional. We measure the computation time at each step for the proposed wavelet as well as Bertram's one when reconstructing the model from level 0 to level 6 . The comparison of the computation times for the two wavelets is shown in Figure 7(b). In general, the wavelet synthesis or analysis algorithm proposed in the paper 
needs a computation time about $1 / 3$ more than Bertram's one, which on the other hand brings better stability and fitting quality.

The analysis effects of three actual highly-detailed models are shown in Figures 8, 9 and 10, respectively, where all resulting meshes at each level are subdivided again to the highest level without considering wavelet coefficients to show their fitting quality relative to the original models. Note that the model meshes are required to have the subdivision connectivity of Loop subdivision, i.e. 1-to-4 triangle splitting. Such subdivision connectivity can be obtained conveniently by some remeshing algorithms (Lee et al., 1998; Eck et al., 1995). On the surfaces of the original models, we have rendered the borderlines of each triangular region corresponding to the coarsest mesh so as to make the connectivity of the meshes clear. The computation times of the wavelet analyses and the numbers of triangles at each level are listed in Table 1 for the three models in detail. These examples have shown sufficient stability and good fitting quality of our wavelet transforms. Progressive transmission of highly-detailed geometric models on a network will be a direct application of the wavelet proposed in the paper. Other applications of the proposed wavelet include shape approximation, data compression, multiresolution rendering, etc.

Table 1. Computation times (seconds) in wavelet analyses

\begin{tabular}{|c|c|c|c|c|c|c|}
\hline \multirow{2}{*}{ Level } & \multicolumn{2}{|c|}{ Venus } & \multicolumn{2}{c|}{ Horse } & \multicolumn{2}{c|}{ Feline } \\
\cline { 2 - 7 } & Triangle Num. & Cost time & Triangle Num. & Cost time & Triangle Num. & Cost time \\
\hline 6 & 1589248 & - & 901120 & - & 2064384 & - \\
\hline 5 & 397312 & 1.337149 & 225280 & 0.759805 & 516096 & 1.737340 \\
\hline 4 & 99328 & 0.367125 & 56320 & 0.206454 & 129024 & 0.474483 \\
\hline 3 & 24832 & 0.106602 & 14080 & 0.059295 & 32256 & 0.138144 \\
\hline 2 & 6208 & 0.033035 & 3520 & 0.018766 & 8064 & 0.043531 \\
\hline 1 & 1552 & 0.037539 & 880 & 0.021010 & 2016 & 0.048779 \\
\hline 0 & 388 & 0.017256 & 220 & 0.008990 & 504 & 0.022508 \\
\hline
\end{tabular}

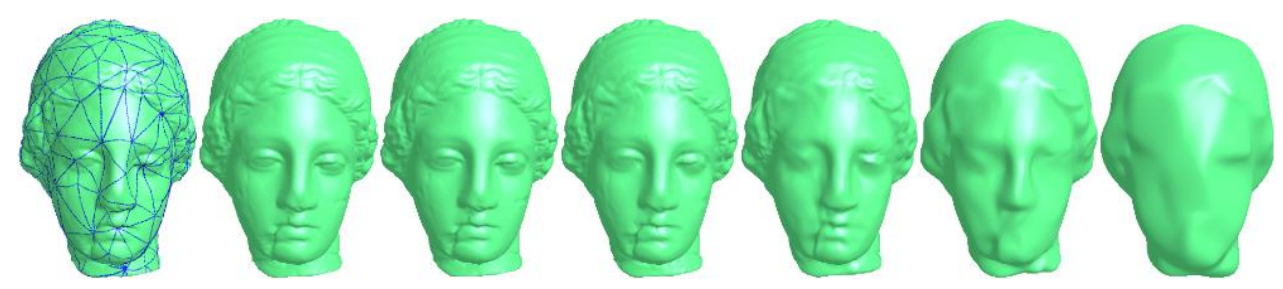

Figure 8. Wavelet analysis for the Venus model

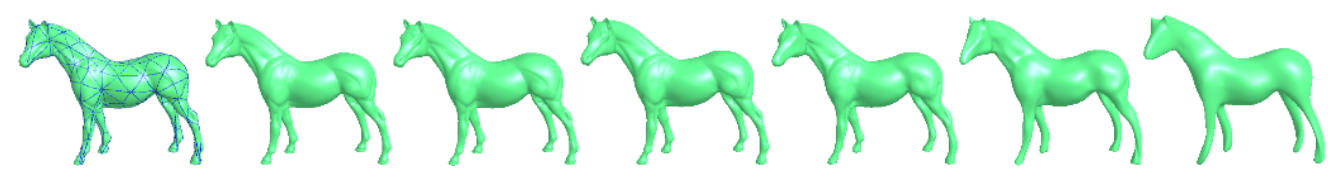

Figure 9. Wavelet analysis for the horse model

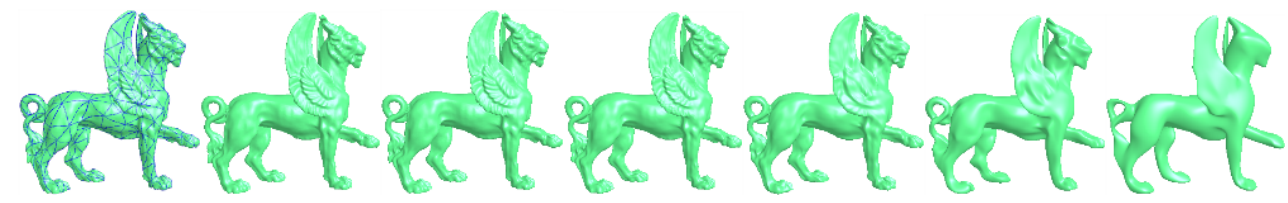

Figure 10. Wavelet analysis for the feline model 


\section{CONCLUSIONS}

In this paper, we have proposed a new butterfly-orthogonal Loop subdivision wavelet based on the lifting scheme. We use a butterfly-style local orthogonalization structure to optimize the free parameters introduced in the wavelet transforms so as to obtain a very stable and nearly semi-orthogonal wavelet. Although it takes a little more computation time than the existing Loop subdivision wavelets, the proposed wavelet possesses a better fitting quality as well as performance of denoising, as demonstrated in the experiments. Similar to the other Loop subdivision wavelets, the butterfly-orthogonal wavelet has a linear computing complexity, because all lifting operations in the wavelet transforms are of perfect in-place computing and relate to only local vertex points or edge points. The proposed wavelet analysis can be used in a wide range of applications, including progressive transmission, shape approximation, data compression, multiresolution rendering and editing of complex models.

\section{ACKNOWLEDGEMENT}

This work was supported by National Key R\&D Program of China (No. 2017YFB0202203), the Defense Industrial Technology Development Program of China (Grant No. C1520110002) and Foundation of Laboratory of Computational Physics.

\section{REFERENCES}

Bertram, M., 2004. Biorthogonal Loop-subdivision wavelets. In Computing, Vol. 72, No. 1-2, pp. 29-39.

Bertram, M. et al., 2004. Generalized B-spline subdivision-surface wavelets for geometry compression. In IEEE Transactions on Visualization and Computer Graphics, Vol. 10, No. 3, pp. 326-338.

Bonneau, G., 1998. Multiresolution analysis on irregular surface meshes. In IEEE Transactions on Visualization and Computer Graphics, Vol. 4, No. 4, pp. 365-378

Catmull, E. and Clark, J., 1978. Recursively Generated B-spline Surfaces on Arbitrary Topological Meshes. In Computer Aided Design, Vol. 10, pp. 350-355.

Doo, D. and Sabin, M., 1978. Behavior of Recursive Division Surfaces near Extraordinary Points. In Computer Aided Design, Vol. 10, pp. 356-360.

Eck, M. et al., 1995. Multiresolution analysis of arbitrary meshes. Proceedings of SIGGRAPH '1995, pp. 173-182.

Khodakovsky, A. et al., 2000. Progressive geometry compression. Proceedings of SIGGRAPH '2000. New Orleans, Louisiana, USA, pp. 271-278.

Lee, A. et al., 1998. MAPS: multiresolution adaptive parameterization of surfaces. Proceedings of SIGGRAPH '1998. Orlando, Florida, USA, pp. 95-104.

Li, D. et al., 2004. Unlifted Loop subdivision wavelets. Proceedings of Pacific Graphics '2004. Seoul, Korea, pp. 25-33.

Loop, C., 1987. Smooth subdivision surfaces based on triangles. M.S. Thesis, Department of Mathematics, University of Utah.

Lounsbery, M. et al., 1997. Multiresolution analysis for surfaces of arbitrary topological type. In ACM Transactions on Graphics, Vol. 16, No. 1, pp. 34-73.

Samavati, F. and Bartels, R., 1999. Multiresolution curve and surface editing: reversing subdivision rules by least-squares data fitting. In Computer Graphics Forum, Vol. 18, No. 2, pp. 97-119.

Samavati, F. et al., 2002. Multiresolution surfaces having arbitrary topologies by a reverse Doo subdivision method. In Computer Graphics Forum, Vol. 21, No. 2, pp. 121-136.

Schröder, P. and Sweldens, W., 1995. Spherical wavelets: efficiently representing functions on the sphere. In Computer Graphics (Proceedings of SIGGRAPH '95), pp. 161-172.

Sweldens, W., 1996. The lifting scheme: a custom-design construction of biorthogonal wavelets. In Appl. Comput. Harmon. Anal., Vol. 3, No. 2, pp. 186-200.

Valette, S. and Prost, R., 2004. Wavelet-based multiresolution analysis of irregular surface meshes. In IEEE Trans. on Visualization \& Computer Graphics, Vol. 10, No. 2, pp. 113-122.

Wang, H. et al., 2006. Efficient wavelet construction with Catmull-Clark subdivision. In Visual Computer, Vol. 22, No. 9-11, pp. 874-884.

Wu, J. and Amaratunga, K., 2003. Wavelet triangulated irregular networks. In International Journal of Geographical Information Science, Vol. 17, No. 3, pp. 273-289.

Zorin, D. et al., 1996. Interpolating subdivision for meshes with arbitrary topology. Proceedings of SIGGRAPH '1996, pp. 189-192. 